\title{
Simulations of Surfactant-Enhanced Spreading
}

\author{
Sean McNamara ${ }^{1,3}$, Joel Koplik ${ }^{1}$, and Jayanth R. Banavar ${ }^{2}$ \\ ${ }^{1}$ Benjamin Levich Institute, \\ City College of New York, \\ New York, NY 10031 \\ ${ }^{2}$ Department of Physics and Center for Materials Physics, \\ Pennsylvania State University, \\ University Park, PA 16802 \\ ${ }^{3}$ Centre Eurpéen de Calcul Atomique et Moléculaire \\ Ecole Normale Supériuere de Lyon \\ 46 , allée d'Italie \\ 69364 Lyon Cedex 07, France
}

\begin{abstract}
We use computer simulation to study the effect of surfactants on a drop spreading on a solid surface. Surfactants enhance spreading, especially when the hydrophobic head of the surfactant molecule is strongly attracted to the solid. A significant part of the spreading is due to the "shielding" of the surface by the surfactant. We use a novel boundary condition that reduces the simulation time by a factor of two.
\end{abstract}

\section{Introduction}

It is an experimental fact that certain surfactants facilitate the spreading of drops on solid surfaces. For certain combinations of surfactants, the increase in spreading is truly significant [1]. We investigate this phenomena using molecular dynamics simulations.

Surfactants could enhance spreading through two mechanisms [2]. First of all, surfactants reduce the surface tension, causing the drop to spread out. A second mechanism is possible if, for example, a drop of water is placed on a greasy surface. A surfactant, such as soap, with a hydrophobic and and a hydrophilic end could interpose itself between the water and the surface, thus shielding the water from the surface. The drop would the spread more easily for the repulsive interactions between the fluid and the surface would be reduced. In this paper, we emphasize the second "shielding" mechanism and show that it is important.

In this paper, we perform several computer "experiments" where a drop is placed on a solid surface, and allowed to spread. We first examine the case without surfactants. The final state of the drop depends delicately on the interaction between the liquid and the solid. We then fix the liquid-solid interaction and experiment with different surfactants, looking for the surfactants which enhance the spreading the most. There are many different parameters, so this paper does not come close to exhausting the possibilities. We investigate three different 
parameters: the relative sizes of the hydrophobic and hydrophilic parts, the solubility of the surfactant and the surfactant-solid interaction. This last parameter is the most significant.

\section{The Experiment}

\section{$2.1 \quad$ Materials}

In our experiments, a drop containing surfactants is placed on a solid surface. Our implementation of surfactants [3] and spreading [4 follows previous work. We define four different types of atoms named $A, B, C$, and $D$. All the necessary components are made of these molecules. A sketch of these components is shown in Fig. 1. The fluid is composed of $A_{2}$ : dimers of atoms of type $A$. We use dimers instead of monomers because dimers are less volatile. The surfactant has the general form $B_{m} C_{n}$. It must be composed of two types of atoms because it has a hydrophilic end and a hydrophobic end. The hydrophilic end is composed of atoms of type $B$ and the hydrophobic end is made of atoms of type $C$. We will use the terms "hydrophobic" and "hydrophilic" to refer to the two ends of the polymers, even though we do not attempt to make the liquid $A_{2}$ mimic water. (Perhaps more appropriate names would be "A-philic" and "A-phobic".) Finally, the solid is composed of atoms of type $D$.

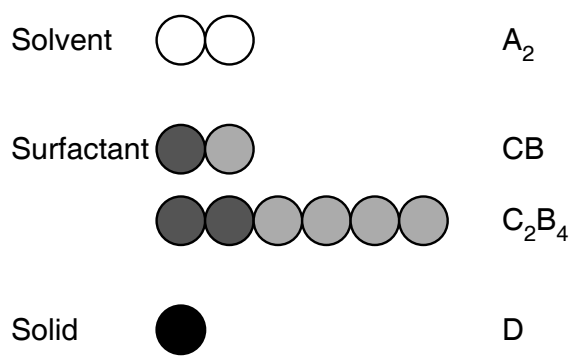

Fig. 1. The different molecules used in the simulations.

The potential between all the atoms is built out of the Lennard-Jones potential [5]:

$$
V_{L J}(r)=4 \epsilon\left[\left(\frac{\sigma}{r}\right)^{-12}-C_{\alpha \beta}\left(\frac{\sigma}{r}\right)^{-6}\right] .
$$

Here $\epsilon$ is the unit of energy, and $\sigma$ is the effective diameter of the atoms. The potential consists of two parts: a strong short-range repulsion (proportional to $r^{-12}$ ), and a longer range attractive potential (proportional to $r^{-6}$ ). We have added the factor $C_{\alpha \beta}$ which depends on the species of the interacting atoms. By changing $C_{\alpha \beta}$, one can increase or decrease the attractive force between the 
molecules. In this paper, $C_{A A}=1$, so $V_{L J}$ reduces to the traditional LennardJones potential. We create hydrophilic and hydrophobic materials by setting $C_{A B}>1$ and $C_{A C}<1 . C_{A D}$ controls the behavior of the liquid on the surface. Roughly speaking, $C_{A D}<1$ gives a liquid which is partially wetting, and $C_{A D}>$ 1 gives a wetting liquid. When $C_{A D}$ is much larger than 1 , terraced spreading is observed. 4

Unless otherwise specified, the interaction coefficients $C_{\alpha \beta}$ have the following values: $C_{A B}=2$ and $C_{A C}=0$, so that the hydrophilic end is very hydrophilic indeed; it attracts fluid atoms more strongly than the fluid atoms attract one another. But between the hydrophobic end and the fluid atoms, there is no long range $\left(r^{-6}\right)$ force, only a short range repulsion. Within the surfact, we set $C_{B B}=1$ and $C_{B C}=C_{C C}=0$. Between the solid and the surfactant we have $C_{B D}=0$ and $C_{C D}=1$. Thus the hydrophobic end of the surfactant is attracted to the solid.

The interaction potential in (11) extends to infinity, which means that every particle directly influences every other particle. In order to solve for the motion of $N$ particles, we would have to calculate $N^{2} / 2$ interactions, which is too expensive. Therefore, we will "cut" the potential at a cutoff radius $r_{c}$, and set $U=0$ for $r>r_{c}$. In this paper, we choose $r_{c}=2.5 \sigma$. We also "shift" the potential so that energy and force are continuous at $r=r_{c}$. The potential becomes

$$
V(r)=\left\{\begin{array}{cc}
V_{L J}(r)-V_{L J}^{\prime}\left(r_{c}\right) r-V_{L J}\left(r_{c}\right), & r<r_{c} \\
0, & r \geq r_{c}
\end{array}\right.
$$

Note that "cutting" and "shifting" the potential changes the properties of the fluid. One must take care when comparing results, because some people shift the potential in different ways, or do not shift it at all. Our method of shifting the potential minimizes integration errors.

Neighboring atoms in molecules do not interact via (2), rather they are bonded together by

$$
V_{\mathrm{bond}}(r)=4 \epsilon\left[\left(\frac{\sigma}{r}\right)^{-12}+\left(\frac{\sigma}{r}\right)^{6}\right] .
$$

Note that the -6 power has been replaced with a +6 . This means that infinite energy is required to break bonds. The -12 power again keeps the atoms separated.

The solid is made by anchoring atoms of type $D$ to a regular array of lattice sites. The $D$ atoms feel a force $\mathbf{F}$ given by

$$
\mathbf{F}=-\left(\frac{\epsilon}{\sigma^{2}}\right) K\left(\mathbf{r}-\mathbf{r}_{0}\right),
$$

where $\mathbf{r}$ is the location of the atom and $\mathbf{r}_{0}$ is the location to which it is tethered. The spring constant $K$ is set to 50 in this paper. The mass of the $D$ atoms is set to 50 so that its resonant frequency will be of order 1 . The other atoms have mass 1 . 


\subsection{Geometry}

As shown in Fig. 2 we will consider the spreading of a cylindrical drops on a flat surface. The surface is a plane perpendicular to the $z$ axis, and the drops axis is parallel to the $y$ axis. The experiment is macroscopically uniform along the $y$ axis. The boundary conditions match this assumption by imposing periodicity in the $y$ direction. In all other directions, a force field prevents particles from escaping from the box. In our experiments, we choose $L_{x}=140 \sigma, L_{y}=12 \sigma$ and $L_{z}=60 \sigma$.

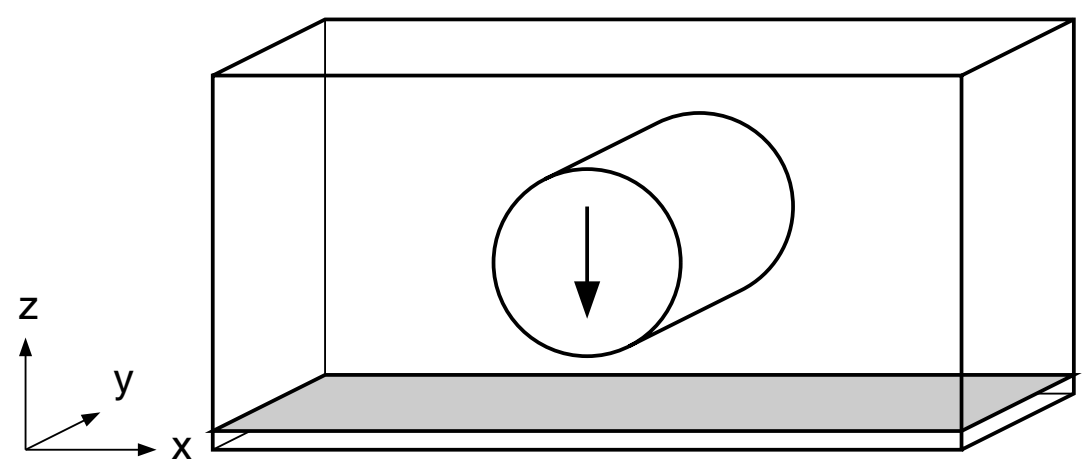

Fig. 2. A sketch of the spreading experiments. Periodic boundary conditions are imposed in the $y$ direction; in all other directions a force field prevents particles from escaping.

In Fig. 3, we show the result of two spreading experiments: one where $C_{A D}=$ 0.8 and another where $C_{A D}=1$. A small change in $C_{A D}$ gives a big change in spreading behavior. When we study surfactants, we will add them to the $C_{A D}=0.8$ fluid, to try to get it to spread like the $C_{A D}=1$ fluid.

Note that the simulations of Fig. 3 have approximate mirror symmetry. We would like to exploit this symmetry to simulate only one half of the spreading drop. To do this, we cut the simulation in half at the midplane, and simulate only the left half, as shown in Fig. $4 \mathrm{a}$. At the midplane, we impose a special kind of mirror image boundary conditions. It turns out that the simplest possible mirror image boundary conditions, where a particle at $(x, y, z)$ has an image at $\left(L_{x}-x, y, z\right)$ does not work. The reason is that when a particle approaches the mirror, it always sees its own image approaching from the other side, and it is repelled by that image. This interaction prevents a half-drop from adhering to its image. What is needed is the "shifted mirror" boundary conditions shown in Fig. $4 \mathrm{~b}$, where a particle's image(s) are shifted by $L_{y} / 2$. In this way, a particle never interacts directly with its own image, and a cluster of particles placed near the mirror boundary spontaneously forms a half drop. 

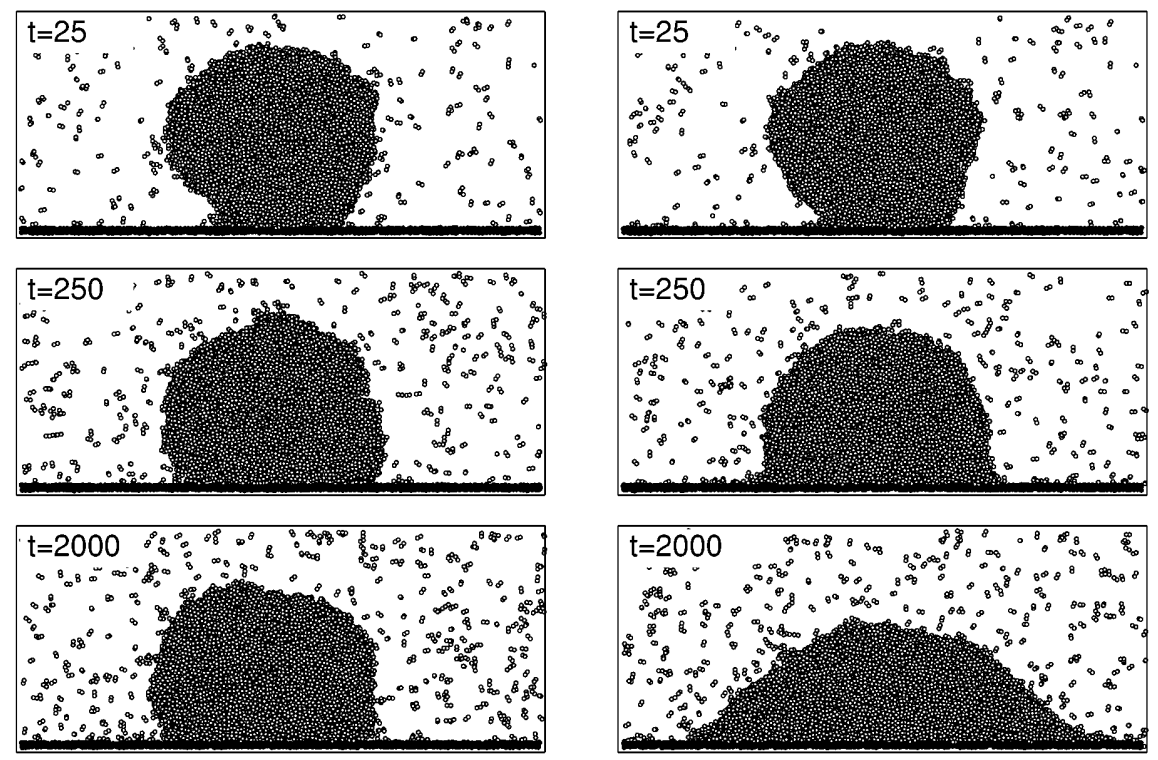

Fig. 3. Results of spreading experiments, with pure fluid: $C_{A D}=0.8$ (left) and $C_{A D}=$ 1 (right). These simulations involve 9000 molecules of $A_{2}$ and 1656 solid atoms.

a)

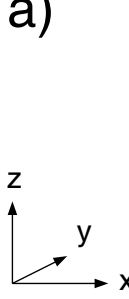

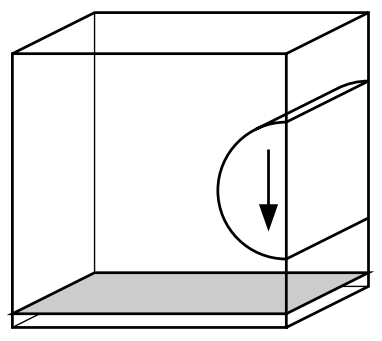

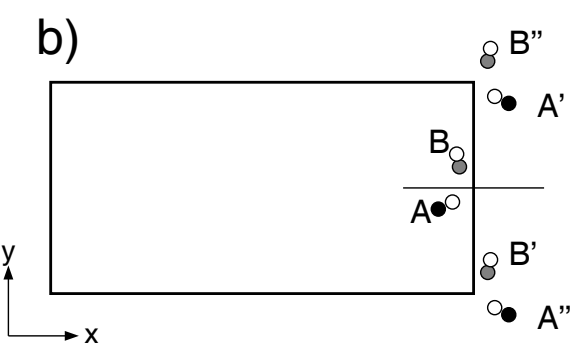

Fig. 4. Boundary conditions for half-drop spreading experiments: a) a sketch of the half-drop experiment; b) the "shifted mirror" boundary conditions applied at the right hand wall (note that the $z$ axis points out of the page in this panel). 
In Fig. 5, we compare the results of simulations shown in Fig. 3 with two equivalent simulations using the shifted mirror boundary conditions. The results show that, within fluctuations, the two boundary conditions are equivalent. All the rest of the simulations presented in this paper use shifted mirror boundary conditions, since they run twice as fast as full simulations.

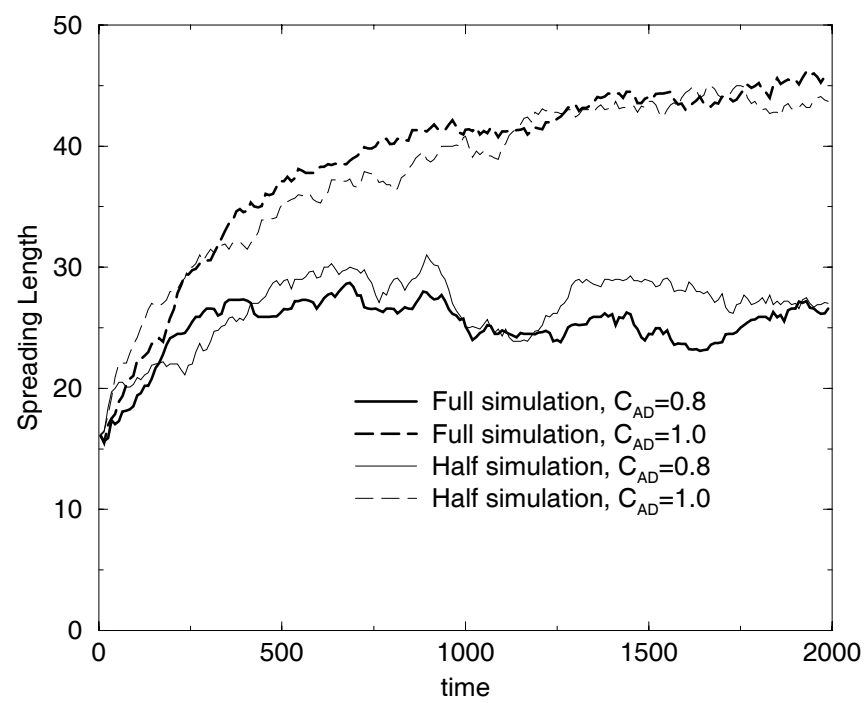

Fig. 5. Comparison of half drop and full drop simulations. The "spreading length" is the distance from the edge of the drop to its midpoint. IT is measured by inspecting the density of fluid atoms just above the solid surface.

\section{The Effect of Surfactants}

We next consider the effect of surfactant composition on spreading. A half drop of 4500 molecules of $A_{2}$ fluid was prepared and 90 molecules of $C_{3} B_{3}, C_{2} B_{4}$ or $C B_{5}$ were added. We placed each drop on a solid surface, and measured its spreading. In Fig. 6 a, we compare the results against pure fluid drops with the same mass ( 4770 molecules of $A_{2}$ ). The surfactants do enhance the spreading, but not by much. Furthermore, it is not clear which surfactant works best. Looking at a snapshot of the simulation, we see that the surfactants are concentrated both at the free surface and at the solid-liquid interface, as shown in Fig. 6b.

The surfactants do partially shield the solvent from the surface, but they also interpose the hydrophobic heads between the solvent and the surface. The 

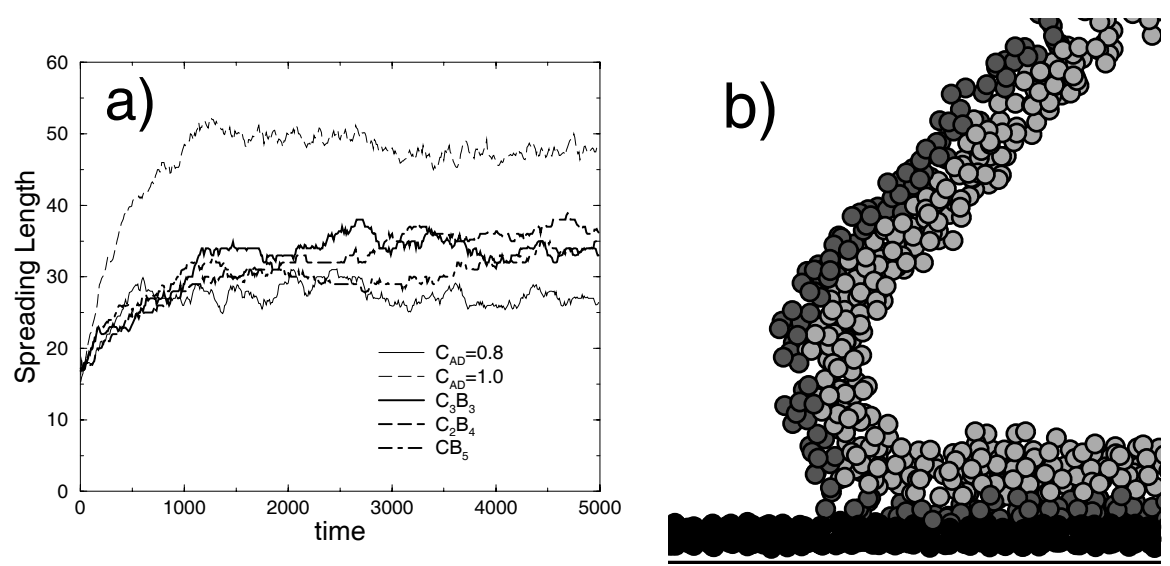

Fig. 6. a) Comparison of different surfactants. The thin lines give pure fluid spreading for two different values of $C_{A D}$. The surfactant drops have $C_{A D}=0.8$; the presence of surfactants does enhance spreading, but not as much as increasing $C_{A D}$ to 1 . The interaction coefficients are as given in Sec. 2.1 $C_{A A}=C_{B B}=C_{D D}=C_{C D}=1$, $C_{A C}=C_{B D}=C_{C C}=0$, and $C_{A B}=2$. b) A magnified view of the edge of the spreading drop at the end of the $C_{2} B_{4}$ simulation in a). The fluid atoms are not shown.

presence of the hydrophobic heads at the liquid-solid boundary reduces spreading because the hydrophobic heads repel the liquid.

Making the surfactant head less hydrophobic might improve the spreading, because the hydrophobic heads at the solid-liquid boundary would be less repulsive to the liquid. We changed the fluid-surfactant interaction parameters to $C_{A C}=0.5$ and $C_{A B}=1.5$, but left all the others unchanged. As a result, the surfactant becomes soluble, that is, it is not confined to the exterior of the drop, and some molecules are present in the interior. However, the majority of the surfactant molecules remain at the surface. The comparison of the soluble and insoluble $C_{2} B_{4}$ surfactant is shown in Fig. 7. As one can see, the soluble surfactant is not more successful than the insoluble one.

The failure of the soluble surfactants to further enhance spreading casts doubt on the "shielding" mechanism. A surfactant must have a hydrophobic part, and this part must always be trapped between the fluid and the solid. If the hydrophobic heads prevent successful spreading even when they are only mildly hydrophobic, it is difficult to see how any surfactant will increase spreading. But if the attraction between the hydrophobic part of the surfactant and the solid surface is increased, the spreading increases remarkably, as shown in Fig. 8

In Fig. 8 b, we see the reason for this surprising behavior: the hydrophobic head penetrate into the solid surface. In this way, they are hidden from the fluid atoms, and the spreading of the drop is much enhanced.

Note that the increased spreading is due to the shielding mechanism, not to a reduction in surface tension. The fluid-surfactant interaction has been left 


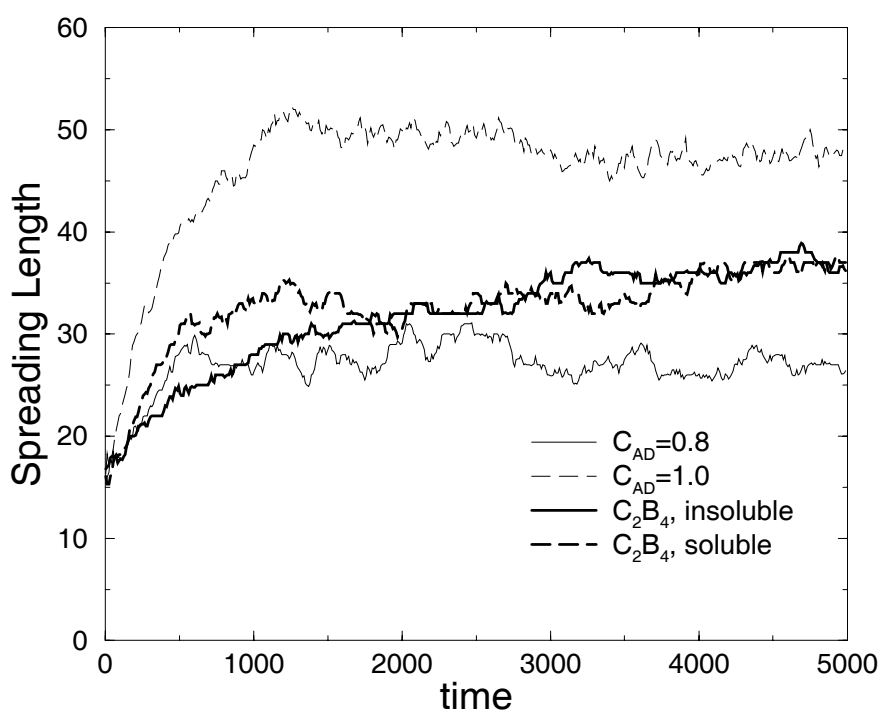

Fig. 7. Comparison between soluble and insoluble surfactants. In both cases, the surfactant is $C_{2} B_{4}$. The insoluble case is taken from Fig. 6] and the interaction coefficients are as stated in Sec. 2.1 The soluble case is the same, except two interaction coefficients have been modified: $C_{A C}=0.5$ and $C_{A B}=1.5$.
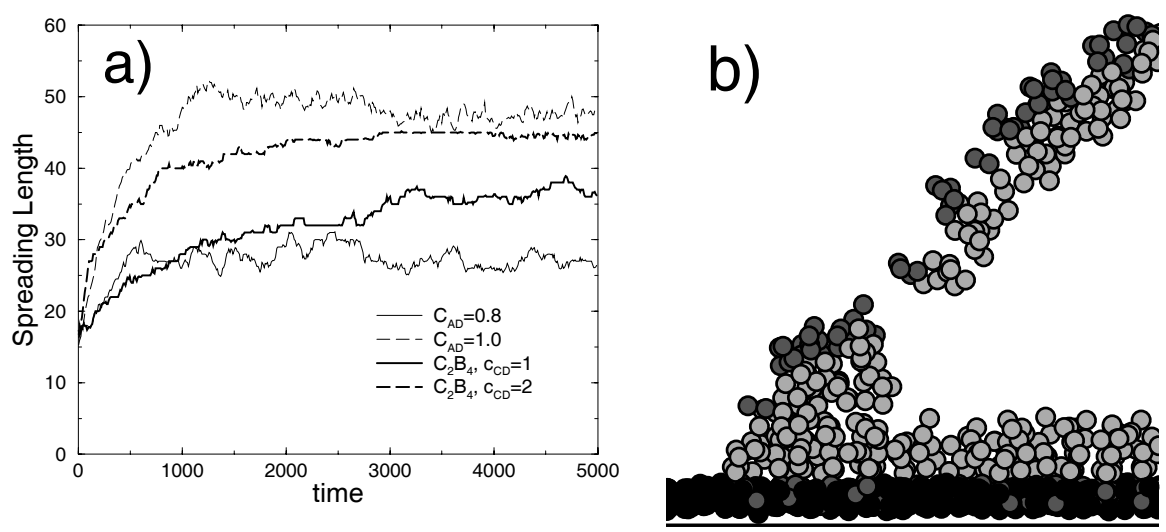

Fig. 8. a) Comparison between the standard surfactants (of Fig. 6) and surfactants with enhanced attraction to the solid. In this last case, $C_{C D}=2$, with all the interaction coefficients as in Sec. 2.1 b) A magnified view of the edge of the spreading drop molecules at the end of the $C_{2} B_{4}$ simulation in a). The fluid atoms are not shown. In contrast to Fig. 6b, the hydrophobic heads penetrate into the solid. This is because the interaction coefficient $C_{C D}$ has been increased from 1 to 2 . 
unchanged, so tension of the free surface will not change. (If anything, the surface tension will increase, because the concentration of surfactant molecules there is lower in Fig. 8b than in Fig. 6b.) We have therefore demonstrated that the shielding mechanism is active.

\section{Conclusions}

We have studied spreading drops using molecular dynamics simulations. We have presented novel boundary conditions which permit us to double the size of the studied drop. We also showed that surfactants enhance spreading. The performance of most surfactants is limited by the fact that the hydrophobic head becomes trapped between the solid and the liquid. Since the hydrophobic head repels the liquid, the liquid soon stops spreading. However, if the attraction between the hydrophobic head and the solid is strong enough, the hydrophobic head buries itself into the solid, thus hiding itself from the liquid, and spreading is greatly enhanced.

Our work suggests that the "shielding" spreading mechanism can be important, if the hydrophobic heads can be hidden from the fluid. In our case, this is acheived by hiding the heads in the solid itself. But more realistic molecules could hide the heads by other mechanisms, such as an orderly array of surfactants at the solid-liquid boundary.

\section{Acknowledgements}

We thank A. Couzis and C. Maldarelli for discussions, the NASA Microgravity Program for financial support, and NCCS and NPACI for computer resources.

\section{References}

1. T. Stoebe, Z. Lin, R. M. Hill, M. D. Ward, and H. T. Davis, Langmuir 12, 337 (1996) and 13, 7282 (1997); M. J. Rosen and L. D. Song, Langmuir 12, 4945 (1996); T. Svitova et al., Langmuir 14, 5023 (1998).

2. A. W. Adamson and A. P. Gast, Physical chemistry of surfaces, 6th ed., (Wiley, New York, 1997).

3. B. Smit, Phys. Rev. A 37, 3431 (1988); F. Schmid, in Computational Methods in Surface and Colloid Science, Surface Science Series 89, 631 (2000).

4. U. D’Ortona, J. De Coninck, J. Koplik, and J. Banavar, Phys. Rev. E, 53562 (1996).

5. M.P. Allen and D.J. Tildesley, Computer Simulation of Liquids, (Oxford, Clarendon Press, 1987). 